\title{
Opinión de los médicos españoles sobre el síndrome de intestino irritable: resultados de un estudio utilizando el método Delphi
}

\author{
C. Almansa, E. Rey, E. Bolaños ${ }^{1}$, M. Palmaㄹ, A. Álvarez Sánchez y M. Díaz-Rubio \\ Servicio de Aparato Digestivo. Hospital Clínico San Carlos. 'Agencia de Evaluación de Tecnologías. Instituto Carlos \\ III. Madrid
}

\section{RESUMEN}

Introducción: el síndrome del intestino irritable (SII) es una patología controvertida, donde ante la escasez de evidencia científica las recomendaciones sobre su manejo clínico y su definición vienen determinadas por métodos de consenso de expertos.

Objetivo: obtener la opinión de los médicos de nuestro país implicados en el manejo de pacientes con SII y conocer los puntos de acuerdo en las principales cuestiones clínicas que plantea.

Material y métodos: se realizó un estudio Delphi a nivel nacional mediante dos envíos a 100 médicos de atención primaria y gastroenterólogos. El cuestionario incluía preguntas sobre el concepto, diagnóstico y tratamiento del SII, evaluando el grado de acuerdo entre los participantes.

Resultados: el 55\% de los encuestados completó los dos envíos. Se obtuvo acuerdo respecto a los síntomas que caracterizan al SII y los de alarma. No existió una actitud clara ante el paciente sin síntomas de alarma, pero sí acuerdo respecto a la realización de colonoscopia en su presencia. Se señaló la necesidad de realizar estudios complementarios en mayores de 40 años. En el tratamiento no encontramos una actitud uniforme, aunque parece existir una orientación diferente según el síntoma predominante.

Conclusiones: los médicos participantes en el estudio demuestran a través de sus respuestas un buen conocimiento conceptual del SII, si bien las discrepancias observadas en las cuestiones sobre diagnóstico y tratamiento demuestran la complejidad y falta de uniformidad que existe en el manejo diario de este síndrome.

Palabras clave: Síndrome del intestino irritable. Estudio Delphi.

\begin{abstract}
Introduction: lack of evidence in most clinical situations re garding irritable bowel syndrome (IBS) enhances the importance of an expert's opinion, which will guide management and even the concept underlying the disease.

Objective: to delve into the knowledge and degree of agreement on main clinical skills for this syndrome among physicians involved in its management.

Material and method: two rounds of a Delphi survey were conducted on 100 physicians: general practitioners (GPs) and gastroenterologists. The questionnaire evaluated agreement among participants in some aspects regarding the definition, diagnosis, and treatment of IBS.

Results: fifty-five percent of participants completed the tworound survey. Agreement was achieved regarding the definition of typical symptoms and red flags characterizing IBS. Although there was no consensus regarding the appropriate management of patients without alarm symptoms, the performance of a colonoscopy on any patient presenting red flags was suggested. Patients were thought to require a wider examination when older than 40. A well defined line of IBS treatment was not found, albeit most physicians tended to choose it depending on the main complaint.

Conclusion: interviewed physicians showed adequate theoretical knowledge of IBS, but lack of uniformity on diagnosis and treatment approach reflects the controversial day-by-day management of this syndrome.
\end{abstract}

Key words: Irritable bowel syndrome. Delphi survey.

Almansa Menchero C, Rey E, Bolaños E, Palma M, Álvarez Sánchez A, Díaz-Rubio M. Opinión de los médicos españoles sobre el síndrome de intestino irritable: resultados de un estudio utilizando el método Delphi. Rev Esp Enferm Dig 2007; 99: $210-217$.

Este estudio ha sido financiado por la Fundación de Investigación Médica Mutua Madrileña.

Recibido: 25-09-06.

Aceptado: 21-12-06.

Correspondencia: Manuel Díaz-Rubio. Servicio de Aparato Digestivo. Hospital Clinico San Carlos. C/ Martín Lagos, s/n. 28040 Madrid.

\section{INTRODUCCIÓN}

El síndrome de intestino irritable (SII) es un trastorno funcional digestivo que sufre hasta el $12 \%$ de la población española, según el criterio utilizado para definirlo (1). Representa el 3\% de las consultas en atención primaria y hasta un $25 \%$ en atención especializada (2) y tiene una amplia repercusión tanto a nivel personal por su im- 
pacto sobre la calidad de vida, como a nivel socio-económico, por los gastos directos y el absentismo laboral que genera (3-5).

Las recomendaciones actuales para su diagnóstico y tratamiento tienen un nivel de evidencia científica en general bajo (6), dado que hay pocos estudios bien diseñados que puedan responder a las preguntas habituales que plantea la práctica clínica diaria.

En este síndrome, las recomendaciones de expertos tienen un gran peso en la literatura científica, empezando por su definición, que es obtenida por métodos de consenso de expertos (7). Sin embargo, el impacto de estas recomendaciones en la práctica diaria es probablemente menor (8).

En un marco donde la medicina basada en la evidencia es difícilmente aplicable por la escasez de evidencias y gran parte de las actitudes están dominadas por la medicina basada en la experiencia (entendiendo experiencia como la cualidad que le hace ser a una persona experta), resulta importante conocer la opinión de los médicos directamente implicados en el manejo del SII (medicina basada en la práctica).

Para conocer esta opinión pueden utilizarse métodos de investigación por encuesta como el método Delphi. Este método ofrece algunas ventajas sobre otros métodos de consenso, considerándose una herramienta especialmente adecuada en situaciones en las que existe poca evidencia empírica y hay, entre otros, factores decisivos de orden subjetivo. Este método además evita problemas asociados con las reuniones de consenso como la escasa representatividad geográfica y la dominancia de ciertas personas en el proceso de comunicación; al ser un método de interacción controlada ninguno de los miembros del panel identifica las opiniones de otros, lo que redunda en una mayor libertad a la hora de expresar las opiniones personales (9-11).

El objetivo del presente estudio es obtener la opinión de los médicos directamente implicados en el cuidado de pacientes con síndrome de intestino irritable (médicos de atención primaria y especialistas de aparato digestivo) y conocer cuáles son los puntos de acuerdo en las principales cuestiones clínicas que plantea.

\section{MATERIAL Y MÉTODOS}

El método utilizado para obtener la opinión de los médicos ha sido un estudio tipo Delphi, a través de dos envíos de un cuestionario realizado especialmente para ello.

\section{Cuestionario}

La generación del cuestionario ha seguido un método sistemático definido a priori. El primer borrador del cuestionario fue diseñado considerando que debía cubrir en sus preguntas, aspectos clínicos del SII (síntomas característicos), datos que pueden modificar la conducta diagnóstica (aspectos temporales, síntomas de alarma, antecedentes de los pacientes, criterios diagnósticos y clasificaciones propuestas en la literatura) estrategia diagnostica utilizada (solicitud de pruebas en diferentes escenarios clínicos) y manejo terapéutico (fármacos y seguimiento). Se utilizaron cuatro formulaciones básicas de preguntas: preguntas con dos respuestas posibles (sí/no), preguntas con tres respuestas posibles (sí, no, a veces o no lo sé), con cinco respuestas posibles (totalmente en descuerdo, algo en desacuerdo, algo de acuerdo, de acuerdo y totalmente de acuerdo) y preguntas con respuesta numérica. Asimismo, se incluyeron varias cuestiones abiertas al objeto de que los entrevistados pudieran aportar información no recogida en las preguntas cerradas. Este primer borrador fue sometido a evaluación por un grupo de consultores, obteniéndose con todas las aportaciones el segundo borrador.

A continuación se realizó una evaluación piloto del segundo borrador. Para esta evaluación se seleccionaron dos centros, uno de atención primaria y otro de atención especializada y se presentó en ambos el proyecto. Se proporcionó el cuestionario a 10 médicos de atención primaria y a 10 especialistas de aparato digestivo de estos centros, que contestaron el cuestionario y nos comunicaron tanto sus impresiones como sus sugerencias de modificación. El grupo de investigación consideró las sugerencias y propuestas de modificación aportadas para redactar el tercer borrador.

Para la generación del cuestionario definitivo, se remitió el último borrador a un grupo de consultores que tras su análisis aportaron nuevas propuestas. Se realizó una valoración de las mismas, redactándose finalmente el cuestionario definitivo.

\section{Identificación de participantes}

El número previsto de participantes fue de 100, 50 médicos de atención primaria y 50 especialistas en aparato digestivo. Para la identificación de posibles participantes se utilizaron dos fuentes. La fuente primaria fue la utilización de bases de datos: para la identificación de médicos de atención primaria se utilizó la base de datos de los participantes en tres cursos de formación en aparato digestivo para médicos de atención primaria realizados anualmente entre 2001 y 2004 (el temario de estos cursos no incluía el síndrome de intestino irritable) y para la identificación de gastroenterólogos se utilizó un listado de especialistas de aparato digestivo. En aquellas Comunidades Autónomas infrarrepresentadas se realizó un contacto aleatorio con centros de atención primaria y servicios de aparato digestivo (a partir de un directorio de centros de salud y de atención especializada) y se solicitó la participación a sus miembros. 


\section{Selección de participantes}

Se realizó un contacto telefónico con todos los posibles participantes identificados para conocer la disponibilidad y aceptación para participar. Aquellos que aceptaron eligieron la forma de contacto que consideraron más adecuada (e-mail, fax, correo ordinario).

\section{Envíos}

Si después de 3 semanas de haber remitido el cuestionario a un contacto no se había obtenido respuesta, se volvía a mandar el cuestionario con una nota recordatoria, realizándose hasta 3 recontactos antes de descartar al participante. En la segunda vuelta se añadieron al cuestionario los resultados obtenidos en la primera y se recortaron algunos ítems, para facilitar la respuesta.

\section{Análisis}

Para el análisis de resultados se obtuvo la frecuencia de respuestas en todos los formatos de pregunta, excepto en las numéricas, donde se calculó la media y el rango intercuartílico. En el caso de las preguntas con 5 posibles opciones se calculó además la media y la mediana de las respuestas.

\section{Criterios de acuerdo}

Se establecieron a priori como criterios de acuerdo los siguientes:

-Preguntas dicotómicas: frecuencia de respuesta mayor al $75 \%$ en un sentido.

-Preguntas con 3 respuestas: frecuencia de respuesta mayor al $66 \%$ en algún sentido.

-Preguntas con 5 respuestas: frecuencia de respuesta inferior al $25 \%$ en sentido contrario, mediana de 4 o mayor; además, se transformaron las respuesta en variables ficticias (con valores de 1-5) y se requirió una media superior a 3,75.

\section{RESULTADOS}

\section{Tasa de respuesta}

De los 50 médicos de atención primaria participantes iniciales se obtuvo la respuesta de 36 en la primera ronda (72\%) y de 27 tras la segunda (54\%). De los 50 especialistas en aparato digestivo participantes iniciales se obtuvo la respuesta de 31 en la primera vuelta y de 28 en la segunda (56\%). En conjunto, la respuesta final fue del $55 \%$, formada por un $49 \%$ de médicos de atención primaria y un $51 \%$ de especialistas de aparato digestivo, sin que existieran diferencias entre los que respondieron y los que no en sexo, edad y años de experiencia.

\section{Distribución geográfica y experiencia clínica}

La distribución geográfica inicial y la proporción final de respuestas de los participantes se muestran en la tabla I. Con respecto a la distribución de los años de experiencia clínica, de los médicos de atención primaria el 14,8\% tenía 5 años o menos de experiencia, el 33,3\% entre 6 y 15 años de experiencia, el $48,1 \%$ entre 15 y 30 años de experiencia y el 3,7\% más de 30 años de experiencia. Entre los especialistas de aparato digestivo, el 21,4\% tenía 5 años o menos de experiencia, el 39,3\% entre 6 y 15 años de experiencia, el $25 \%$ entre 15 y 30 años de experiencia y el 14,3\% más de 30 años de experiencia. Con respecto a la distribución por sexos, el 32,7\% del total eran mujeres.

Tabla I. Distribución geográfica de los participantes y tasa de respuesta

\begin{tabular}{lcc}
\hline CC.AA. & Número inicial & Tasa de respuesta \\
\hline Andalucía & 10 & $5(50 \%)$ \\
Aragón & 6 & $3(50 \%)$ \\
Asturias & 6 & $3(50 \%)$ \\
Baleares & 2 & $0(0 \%)$ \\
Canarias & 2 & $1(50 \%)$ \\
Cataluña & 18 & $12(66 \%)$ \\
Castilla La Mancha & 10 & $5(50 \%)$ \\
Castilla León & 8 & $4(50 \%)$ \\
Extremadura & 4 & $2(50 \%)$ \\
Galicia & 4 & $3(75 \%)$ \\
Madrid & 18 & $11(61 \%)$ \\
País Vasco & 4 & $3(75 \%)$ \\
Valencia & 8 & $3(37 \%)$ \\
\hline
\end{tabular}

\section{Síntomas característicos del SII}

Se preguntó a los participantes acerca de aquellos síntomas que consideraban característicos del SII: el dolor abdominal, el dolor hipogástrico, la alteración de la consistencia de las heces, el aumento o disminución de la frecuencia defecatoria con el dolor y la distensión alcanzaban los niveles requeridos para considerar acuerdo. La tabla II recoge los resultados sobre todos los síntomas propuestos.

\section{Síntomas de alarma}

En el cuestionario se incluían preguntas para valorar la importancia de los síntomas de alarma, tanto en relación con su presencia como con su ausencia. Con respecto a lo primero, el $96 \%$ de los participantes consideró que la presencia de síntomas de alarma obliga a considerar otros diagnósticos y por tanto a realizar estudios complementarios. Con respecto a las implicaciones de su ausencia, el $58 \%$ consideraron que su ausencia en el marco clínico adecuado no es suficiente para afirmar el diagnóstico.

La tabla III recoge los resultados de qué síntomas se deberían considerar como síntomas de alarma. 
Tabla II. Síntomas característicos de SII

\begin{tabular}{lcccc}
\hline & \% en desacuerdo & Mediana & Media & Acuerdo \\
\hline Dolor abdominal & $0 \%$ & 4,01 & 4 & Sí \\
Dolor epigástrico & $50,9 \%$ & 2,54 & 2 & No \\
Dolor hipogástrico & $1,8 \%$ & 3,94 & 4 & Sí \\
Alivia defecar & $0 \%$ & 4,25 & 4 & Sí \\
Alivia ventosear & $3,6 \%$ & 3,92 & 4 & Sí \\
Aumenta con ingesta & $70,9 \%$ & 2,36 & 2 & No \\
Aumento frecuencia & $2 \%$ & 3,93 & 4 & Sí \\
Disminución frecuencia & $9 \%$ & 3,51 & 4 & Sí \\
Cambio consistencia & $0 \%$ & 3,95 & 4 & Sí \\
Esfuerzo defecatorio & $7 \%$ & 3,22 & 3 & No \\
Urgencia & $7 \%$ & 3,64 & 4 & Sí \\
Tenesmo & $20 \%$ & 3,18 & 3 & No \\
\hline Accordo
\end{tabular}

Acuerdo si mediana $\geq 4$ y/o media $\geq 3,75$

Tabla III. Síntomas de alarma

\begin{tabular}{lcccc}
\hline & \% en desacuerdo & Mediana & Media & Acuerdo \\
\hline Anorexia & $0 \%$ & 4 & 4,04 & Sí \\
Astenia & $18 \%$ & 3,2 & 3 & No \\
Fiebre & $4 \%$ & 4 & 4,22 & Sí \\
Pérdida de peso & $0 \%$ & 5 & 4,63 & Sí \\
Rectorragia & $0 \%$ & 5 & 4,65 & Sí \\
Diarrea nocturna & $4 \%$ & 4 & 3,98 & Sí \\
Heces acintadas & $55 \%$ & 2,55 & 2 & No \\
Vómitos & $16 \%$ & 3,05 & 3 & No \\
Tenesmo & $24 \%$ & 2,94 & 3 & No \\
Distensión & $78 \%$ & 2,18 & 2 & No \\
\hline
\end{tabular}

Acuerdo si mediana $\geq 4$ y/o media $\geq 3,75$.

\section{Aspectos cronológicos y utilidad de criterios diagnósticos}

El 96\% de los participantes consideraron que el tiempo de evolución de los síntomas es un factor importante en el diagnóstico del SII, planteándose el $87 \%$ de ellos un diagnóstico diferente si el tiempo de evolución de los mismos es inferior a 3 meses. En general los participantes consideran necesario un tiempo de evolución de los síntomas en torno a 8 meses (media $8,48 \pm 4,78$, mediana $7,5)$ para realizar un diagnóstico de SII.

Con respecto a los criterios clínicos para diagnosticar el SII, el 70\% de los médicos participantes está de acuerdo en que facilitan el diagnóstico de SII y sólo un 2\% se muestra en desacuerdo. A la pregunta de qué criterios conocen y usan en su práctica habitual, más de un $75 \%$ dice conocer y utilizar al menos a veces los criterios de Roma II, frente a menos de un $25 \%$ que ocasionalmente emplean los criterios de Manning.

Un $96 \%$ de los encuestados conoce también la clasificación por grupos sintomáticos (diarrea, estreñimiento o ritmo alternante) y un $76 \%$ la utiliza habitualmente. La mayoría están de acuerdo en considerarla una clasificación apropiada (4\% de respuestas en desacuerdo) y hasta un $65 \%$ responde que se debe modificar la actitud diagnóstica en función del síntoma predominante.

\section{Características propias del paciente}

No parece existir un claro acuerdo en considerar la edad como un elemento clave para realizar el diagnóstico clínico del SII, existiendo desacuerdo (70\% de respuestas negativas) en considerar que la clínica es suficiente para llegar al diagnóstico de esta entidad en pacientes jóvenes.

Sin embargo, cuando se preguntó sobre cuál se consideraba el limite de edad para diferenciar joven de mayor, los participantes proporcionaron la edad de 40 años (media $40,24 \pm 5,92$, mediana 40 ) y el $100 \%$ de los participantes estimó necesario realizar pruebas diagnósticas complementarias ante un paciente mayor de esta edad, aunque presentase un cuadro clínico característico.

Con respecto a los antecedentes personales se observó acuerdo en realizar un estudio más exhaustivo en aquellos pacientes que presentasen antecedentes familiares de primer grado de cáncer de colon $(100 \%$ de respuestas de acuerdo) o de enfermedad inflamatoria intestinal ( $84 \%$ de respuestas de acuerdo).

\section{Estrategia diagnóstica}

Se propusieron cuestiones en 6 apartados diferentes que venían determinados en función del ritmo intestinal predominante (diarrea, estreñimiento, alternancia) y la ausencia o presencia de síntomas de alarma. Tras el análisis de los resultados del primer envío del cuestionario, en todos los grupos había un claro acuerdo en realizar un análisis de sangre básico (hemograma y bioquímica), por lo que en la segunda vuelta se integró al análisis de sangre como parte del diagnóstico clínico (basado en la historia clínica). La tabla IV recoge los resultados respecto a los estudios diagnósticos propuestos.

\section{Tratamiento}

Más del $90 \%$ de los facultativos considera que en las etapas iniciales del SII ocasionalmente basta con tranquilizar al paciente. Sin embargo, una mayoría de los médi$\cos (85 \%)$ reconoce iniciar a veces tratamiento empírico durante 4-6 semanas, basándose en un 98\% de los casos en el síntoma guía.

Respecto a las medidas terapéuticas y los fármacos concretos, las respuestas fueron extraordinariamente variadas en las combinaciones. Las figuras 1,2 y 3 recogen los resultados de forma global.

Si nos centramos en la primera línea de tratamiento, los participantes señalaron:

-Predominio de diarrea: como primera línea de tratamiento la mayoría coinciden en aconsejar modificaciones del estilo de vida e incrementar la fibra de la dieta, si bien el $51 \%$ de ellos señalan que desde el primer momento se pueden asociar antidiarreicos y/o espasmolíticos a estas medidas. 
Tabla IV. Estrategia diagnóstica propuesta en función de los síntomas

\begin{tabular}{|c|c|c|c|c|c|c|c|c|c|c|c|c|}
\hline & \multicolumn{2}{|c|}{ Diarrea sin alarma } & \multicolumn{2}{|c|}{ Diarrea con alarma } & \multicolumn{2}{|c|}{ Estreñimiento sin alarma } & \multicolumn{2}{|c|}{ Estreñimiento con alarma } & \multicolumn{2}{|c|}{ Alternante sin alarma } & \multicolumn{2}{|c|}{ Alternante con alarma } \\
\hline & Media & Acuerdo & Media & Acuerdo & Media & Acuerdo & Media & Acuerdo & Media & Acuerdo & Media & Acuerdo \\
\hline Diagnóstico clínico & 2,95 & No & 1,70 & No & 2,95 & No & 1,89 & No & 3,00 & No & 1,73 & No \\
\hline RFA & 3,7 & Sí & 4,3 & Sí & 3,1 & No & 3,8 & Sí & 3,4 & No & 4,0 & Sí \\
\hline TSH & 3,6 & No & 4,1 & Sí & 3,2 & No & - & - & 3,4 & No & - & - \\
\hline Marcadores tumorales & - & - & 3,51 & No & - & - & 3,54 & No & - & - & 3,53 & No \\
\hline Coprocultivo & 3,5 & No & 3,6 & No & - & - & - & - & 2,8 & No & 3,3 & No \\
\hline Enema opaco & 2,40 & No & 2,83 & No & 2,85 & No & 2,87 & No & 2,64 & No & 2,89 & No \\
\hline Colonoscopia & 2,8 & No & 4,4 & Sí & 2,85 & No & 4,5 & Sí & 3,0 & No & 4,6 & Sí \\
\hline
\end{tabular}

Acuerdo si media $\geq 3,75$.

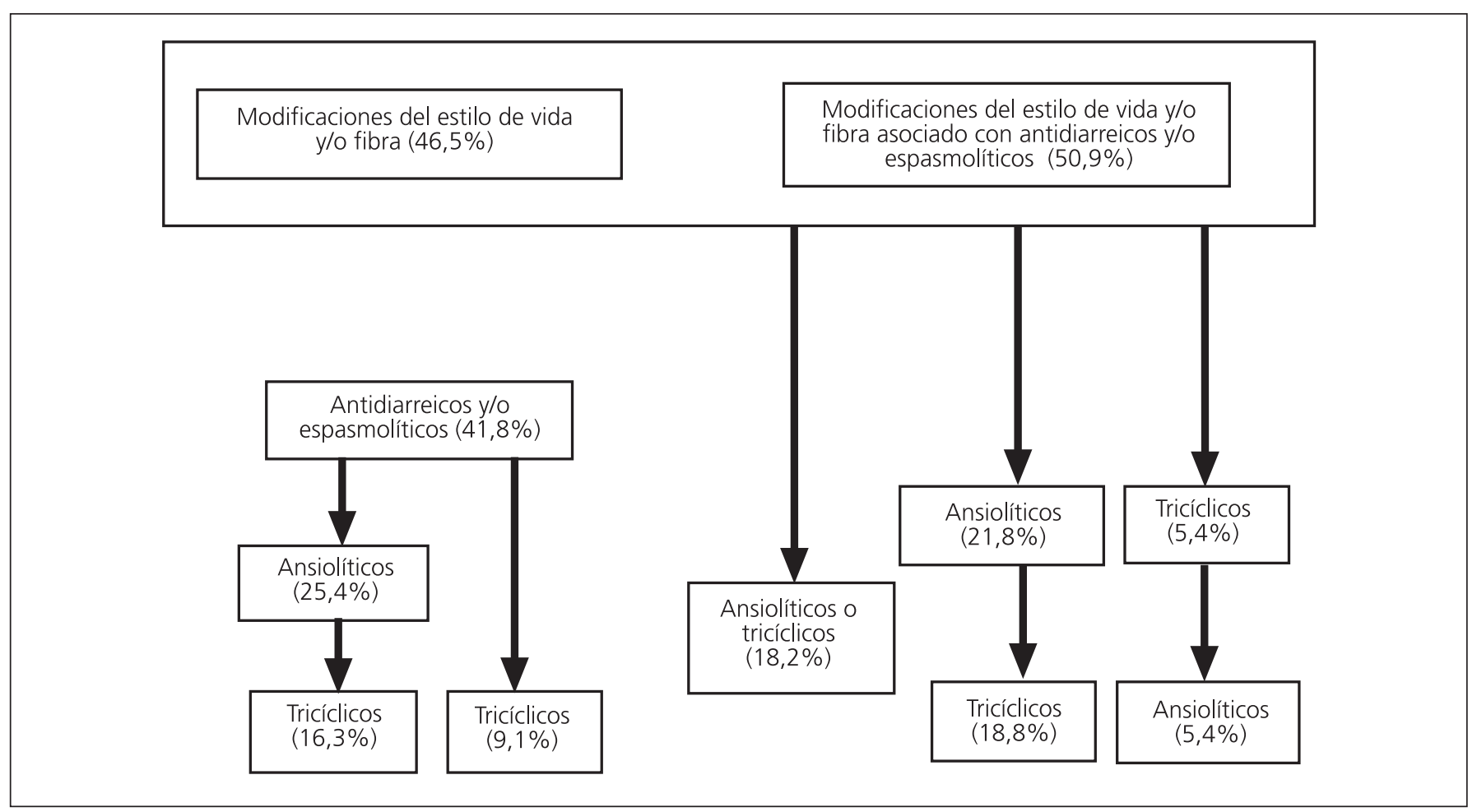

Fig. 1. Manejo terapéutico del SIl con predominio de diarrea.

-Predominio de estreñimiento: el 60\% de los participantes consideran que la primera medida debería ser las modificaciones del estilo de vida y fibra, aunque un $25 \%$ de los encuestados asociarían desde la primera visita laxantes.

-Ritmo alternante: el $80 \%$ de los participantes consideran que inicialmente sólo se deben recomendar cambios del estilo de vida y fibra y un $20 \%$ que se deben asociar espasmolíticos como tratamiento de primera línea.

\section{Seguimiento}

Un $20 \%$ de los participantes señala que el seguimiento se debería realizar a demanda del paciente; el resto señala intervalos fijos para el seguimiento, sugiriéndose una periodicidad semestral. Con respecto a quien debería realizar el seguimiento, un $70 \%$ considera que el médico de atención primaria debe ser siempre responsable del mismo, aunque casi todos los encuestados (96\%) coinciden en que en ocasiones el encargado debe ser el especialista de digestivo.

\section{DISCUSIÓN}

El objetivo de este trabajo fue proporcionar una "fotografía” de la opinión de los médicos españoles respecto al síndrome de intestino irritable, centrándonos en los aspectos clínicamente relevantes. Aunque y pese a insistir 


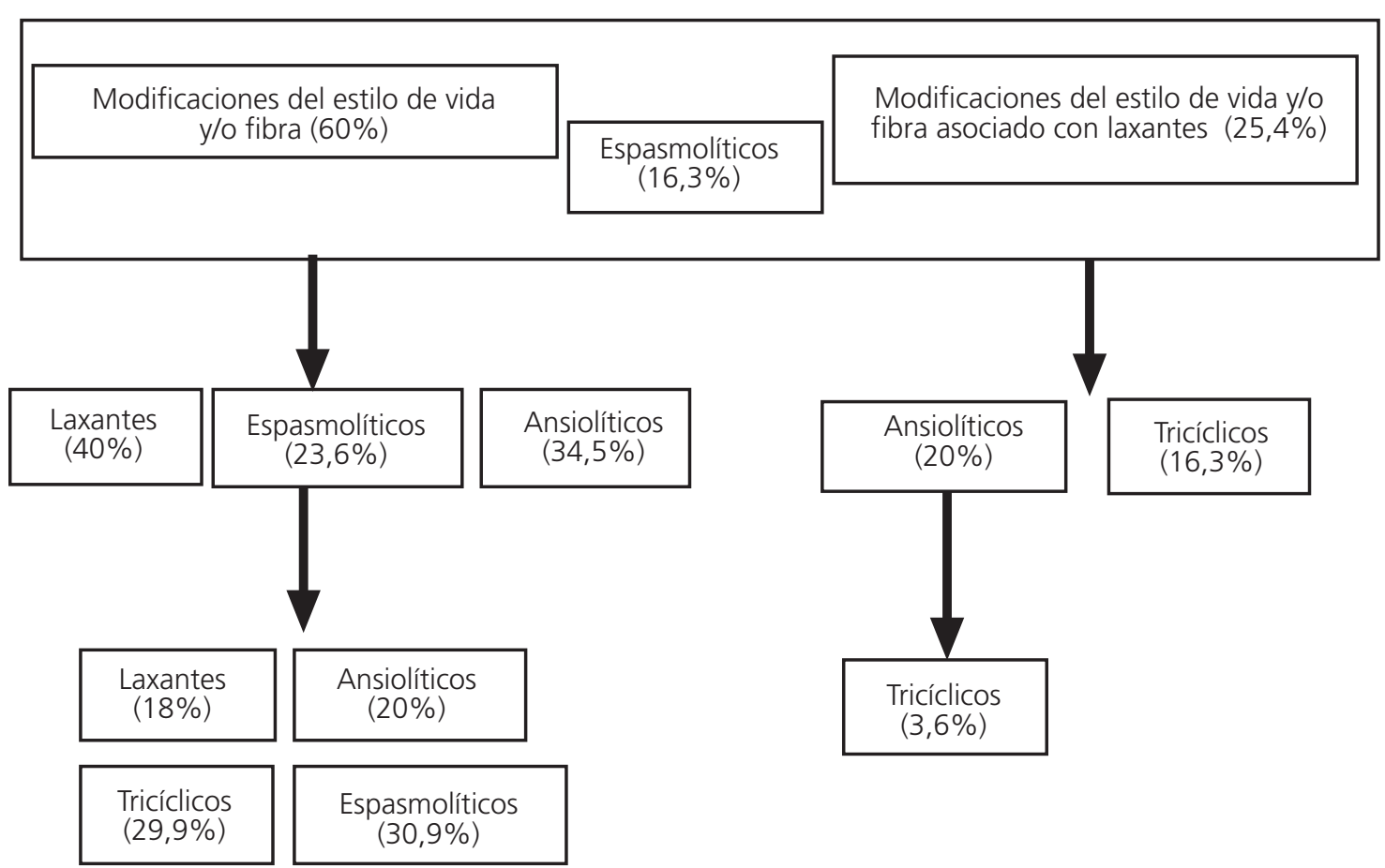

Fig. 2. Manejo terapéutico del SII con predominio de estreñimiento.

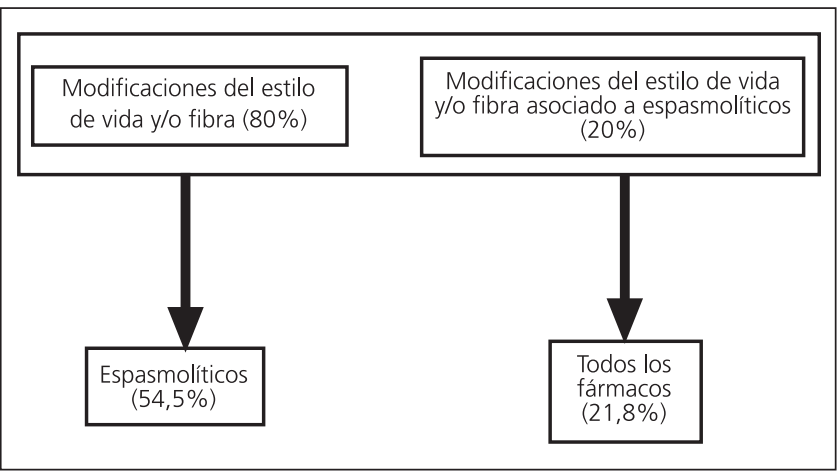

Fig. 3. Manejo terapéutico del SIl con ritmo alternante.

en ello, no se consiguió una respuesta mayor del $60 \%$, no obstante esta es una tasa de respuesta aceptable e incluso superior a la obtenida en estudios con similar metodología (12).

De forma global, el resultado de comparar la opinión de nuestros médicos con la evidencia científica actualmente disponible y las guías clínicas nacionales e internacionales $(6,13,14)$ pone de manifiesto muchas coincidencias, pero también algunas divergencias.

Conceptualmente el SII es casi lo mismo para todos los profesionales entrevistados, dolor abdominal o hipogástrico que alivia al defecar o ventosear, relacionado con un cambio en la consistencia de las heces o frecuencia defecatoria, y asociado también a distensión abdominal, este último hecho es francamente interesante porque si bien no se incluye en la definición establecida por con- senso en Roma II, es una característica propia y bien documentada en el SII (15).

Al igual que en las guías clínicas, los médicos consultados están de acuerdo en considerar necesaria la identificación de síntomas de alarma antes de realizar el diagnóstico clínico de SII, considerando como síntomas de alarma generales anorexia, fiebre y pérdida de peso y digestivos rectorragia y diarrea nocturna; de acuerdo también a lo publicado en la literatura el comienzo abrupto de los síntomas es considerado también como un signo de alarma.

Parece también imprescindible interrogar sobre los antecedentes familiares del enfermo, que en determinados casos, familiar de primer grado con cáncer de colon o enfermedad inflamatoria intestinal nos obligarían a realizar un estudio más exhaustivo del paciente.

Los médicos entrevistados señalan los 40 años como edad límite a partir de la cual es necesario realizar pruebas complementarias para descartar otras patologías más graves, lo que reflejaría una actitud más conservadora (y posiblemente defensiva) de la recomendada en las guías, que si bien no es unánime oscila entre los 45 (6) y los 50 años $(13,14)$; no existe demasiada evidencia científica al respecto y tan sólo hay un estudio disponible, que señala como más apropiada la edad de 50 años (16).

La mayoría de los médicos encuestados consideran que los criterios clínicos facilitan el diagnóstico del SII y más del $75 \%$ de ellos manifiesta conocer y utilizar al menos a veces los criterios de Roma II en su práctica habitual. Estas cifras contrastarían con otras publicadas, ya 
que algunos estudios ponen de manifiesto que en la práctica clínica los médicos, especialmente de atención primaria, utilizan muy poco estos criterios e incluso un porcentaje alto de los mismos los desconocen $(2,17,18)$. Los datos obtenidos no permiten conocer cuál es la razón de esta discrepancia.

La clasificación en subgrupos de síntomas (diarrea, estreñimiento o ritmo alternante) es catalogada como una clasificación razonable por una mayoría de los participantes para dirigir el manejo de estos pacientes. Es llamativo cómo los médicos participantes señalan por término medio necesaria una evolución en torno a 8 meses para considerar el diagnóstico de SII y no se muestran claramente de acuerdo con que los síntomas deban estar presentes al menos un 25\% del tiempo. Ambos datos están en línea con las principales modificaciones que han incluido los recientemente publicados criterios de Roma III respecto a los previos de Roma II (19).

Respecto a la estrategia diagnóstica, parece que estos médicos son partidarios de realizar de entrada un análisis de sangre básico como complemento del diagnóstico clínico del paciente con SII, añadiendo la determinación de TSH y cultivo de heces cuando la diarrea es el síntoma predominante. En caso de existir síntomas de alarma los facultativos encuestados están de acuerdo en solicitar además reactantes de fase aguda y colonoscopia. Si comparamos esta estrategia diagnóstica con la recomendada por los diferentes paneles de expertos, encontramos en general un parecido razonable, aunque existen dos importantes excepciones: en primer lugar, porque nuestros médicos no recomiendan realizar unánimemente despistaje de enfermedad celiaca en los pacientes con diarrea, que estaría plenamente justificado e incluso recomendado por la prevalencia (20) de esta enfermedad en pacientes con síntomas sugerentes de SII, por lo que deducimos que esta no parece ser una actitud habitual en nuestros facultativos. Y en segundo lugar, por la tendencia a recomendar la realización de colonoscopia probablemente como despistaje de cáncer de colon a una edad inferior a la internacionalmente recomendada.

Según la información que proporcionan los médicos, el abordaje terapéutico que realizan en el SII es bastante uniforme. La mayoría coinciden en que a veces el mejor de los tratamientos es tranquilizar al paciente. Como se ha podido comprobar en diferentes ensayos clínicos una buena relación médico-paciente es fundamental en el curso y evolución de esta enfermedad $(6,21)$ y de hecho puede influir notablemente en la conducta de los pacientes en cuanto a consultas médicas (22). Es interesante la confianza que los facultativos tienen en las modificaciones del estilo de vida y la dieta. Si bien y pese al poco fundamento científico se tiende a insistir en la ingesta de fibra independientemente del tipo de síntomas que presenta el paciente. Esto podría reflejar dos cosas, en primer lugar que la mayoría de enfermos que son vistos en nuestras consultas presentan síntomas leves que pueden mejorar sólo con estas medidas y en segundo lugar que nos en- contramos ante una actuación médica basada en una experiencia positiva cuyos resultados podrían explicarse por el efecto placebo, que según datos de la revisión de ensayos clínicos en el SII superaría el 40\% $(23,24)$.

En general el tratamiento farmacológico empírico en el SII no encuentra un acuerdo unánime, si bien existe bastante tendencia a dirigirlo en función de la clínica que presenta el paciente, de forma bastante acorde con las recomendadas en las guías clínicas $(13,14)$, nos parece sin embargo muy llamativa, la recomendación de fármacos espasmolíticos como tratamiento de primera línea en pacientes con SII y predominio de diarrea que sin embargo se considera un fármaco de segunda línea en el tratamiento del resto de pacientes (con estreñimiento y alternantes), sin que sepamos claramente cuál es la razón. Habitualmente las pautas de tratamiento que nuestros facultativos refieren emplear son cortas, pasando después a un escalón terapéutico superior añadiendo fármacos con propiedades ansiolíticas o tranquilizantes coadyuvantes.

En definitiva los médicos consultados tanto de atención primaria como digestivos, muestran un conocimiento adecuado sobre el concepto de SII, y en su relación con la práctica expresan una forma de actuar parcialmente ajustada a lo publicado en las diferentes guías, lo que de alguna manera refleja las dificultades que pueden aparecer en el manejo diario de estos pacientes, donde ocasionalmente y con el objeto de conseguir el mayor beneficio para el enfermo los profesionales de la salud se ven obligados a sustituir la medicina basada en la evidencia por la medicina basada en la experiencia.

\section{AGRADECIMIENTOS}

Agradecemos la participación como consultores de Fermín Mearin (Barcelona), Antonio Caballero (Granada), Julio Ponce (Valencia), Jesús García-Cano (Cuenca) y Cristina Moreno (Madrid) así como a todos los médicos de atención primaria y gastroenterólogos participantes, sin los cuales este proyecto no hubiera sido posible. Agradecemos a Paz Fernee y Ana Linares su inestimable ayuda en la preparacion de la versión inglesa del manuscrito.

La Dra. Cristina Almansa es actualmente receptora de una beca AGA-SEPD. Beca Jon Isenberg, en el Departamento de Gastroenterología de la Clínica Mayo. Jacksonville, EE.UU.

\section{BIBLIOGRAFÍA}

1. Mearin F, Badia X, Balboa A, Baro E, Caldwell E, Cucala M, et al. Irritable bowel syndrome prevalence varies enormously depending on the employed diagnostic criteria: Comparisons of Rome II versus previous criteria in a general population. Scand J Gastroenterol 2001; 36: 1155-61. 
2. Thompson WG, Heaton KW, Smyth GT, Smyth C. Irritable bowel syndrome in general practice: Prevalence, characteristics, and referral. Gut 2000; 46: 78-82.

3. Badia X, Mearin F, Balboa A, Baro E, Caldwell E, Cucala M, et al. Burden of illness in irritable bowel syndrome comparing Rome I and Rome II criteria. Pharmacoeconomics 2002; 20: 749-58.

4. Sandler RS, Everhart JE, Donwitz M, Adams E, Cronin K, Goodman $\mathrm{C}$, et al. The burden of selected digestive diseases in the United States. Gastroenterology 2002; 122: 1500-11.

5. Hungin AP, Whorwell PJ, Tack J, Mearin F. The prevalence, patterns and impact of irritable bowel syndrome: an international survey of 40,000 subjects. Aliment Pharmacol Ther 2003; 17: 643-50.

6. Jones J, Boorman J, Cann P, Forbes A, Gomborone J, Heaton K, et al. British Society of Gastroenterology guidelines for the management of the irritable bowel syndrome. Gut 2000; 47 (Supl. II): ii1-ii9.

7. Drossman DA, Corazziari E, Talley NJ, Thompson WG, Whitehead WE. Rome II: the functional gastrointestinal disorders. Diagnosis, pathophysiology and treatment: a multinational consensus. 2nd ed. McLean, Va: Degnon Associates; 2000.

8. Yawn BP, Lydick E, Locke GR, Wollan PC, Bertran SL, Kurland MJ. Do published guidelines for evaluation of irritable bowel syndrome reflect practice? BMC Gastroenterology 2001, I: II.

9. Verhagen AP, De Vet HC, De Brie RA, Kessels AG, Boers M, Bouter LM, et al. The Delphi list a criteria list for quality assessment of randomized clinical trials for conducting systematic reviews developed by Delphi consensus. J Clin Epidemiol 1998, 51: 1235-41.

10. Powell C. The delphi technique: myths and realities. J Adv Nurs 2003; 41: 376-82.

11. Mokkink LB, Terwee CB, Knol DL, Stratford DW, Alonso J, Patrick DL, et al. Protocol of the COSMIN study: Consensus based standards for the selection of health measurement instruments. BMC Med Res Methodol 2006; 24: 2.

12. Almasio PL, Niero M, Anglioli D, Ascione A, Gullini S, Minoli G, et al. Experts' opinions on the role of liver biopsy in HCV infection: a Delphi survey by the Italian Association of Hospital Gastroenterologist (A.I.G.O.). J Hepatol 2005; 43(3): 381-7.

13. AGA Technical Review on Irritable Bowel Syndrome. Gastroentero- logy 2002; 123: 2108-31

14. American College of Gastroenterology Functional Gastrointestinal Disorders Task Force. Evidence based position statement on the management of irritable bowel syndrome in North America. AJG 2002; 97: 11(Supl.): S1-S5.

15. Chang L, Lee OY, Naliboff B, Schmulson M, Mayer EA. Sensation of bloating and visible abdominal distension in patients with irritable bowel syndrome. Am J Gastroenterol 2001; 96: 3341-7.

16. Hammer J, Eslick GD, Howell SC, Altiparmak E, Talley NJ. Diagnostic yield of alarm features in irritable bowel syndrome and functional dyspepsia. Gut 2004; 53: 666-72.

17. Gladman LM, Gorard DA. General practitioner and hospital specialist attitudes to functional gastrointestinal disorders: Aliment Pharmacol Ther 2003; 17: 651-4.

18. Lea R, Hopkins V, Hastleton J, Houghton LA, Whorwell PJ. Diagnostic criteria for irritable bowel syndrome: Utility and applicability in clinical practice. Digestion 2004; 70: 210-3.

19. Longstrech GF, Thompson WG, Chey WD, Houghton LA, Mearin F, Spiller RC. Functional bowel disorders. Gastroenterology 2006; 130: 1480-91.

20. Sanders DS, Carter MJ, Hurlstone DP. Association of adult coeliac disease with irritable bowel syndrome: A case-control study in patients fulfilling the ROME II criteria referred to secondary care. Lancet $2001 ; 358: 1504-8$.

21. Owens DM, Nelson DK, Talley NJ. The irritable bowel syndrome: Long term prognosis and the physician patient interaction. Ann Intern Med 1995; 122: 107-12.

22. Izquierdo S, Rey E, Garcia Alonso M, Almansa C, Diaz-Rubio M. Has the identification of rectal hypersensitivity any implication in the clinical outcome of irritable bowel syndrome? Rev Esp Enferm Dig 2005; 97: 223-8.

23. Spiller RC. Problems and challenges in the design of irritable bowel syndrome clinical trials: experience from published trials. Am J Med 1999; 107(5A): 91S-97S.

24. Patel SM, Stason WB, Legedza A, Ock SM, Kaptchuk TJ, Conboy L et al. The placebo effect in irritable bowel syndrome trials: A metaanalysis. Neurogastroenterol Motil 2005; 17: 332-40. 